\title{
Patrones electroforéticos de hemoproteínas salivares (nitroforinas) de Rhodnius colombiensis y Rhodnius prolixus (Hemiptera, Reduviidae, Triatominae)
}

\author{
Andrea Arévalo ${ }^{1}$, Julio César Carranza ${ }^{1}$, Felipe Guhl ${ }^{2}$, Gustavo Adolfo Vallejo ${ }^{1}$ \\ ${ }^{1}$ Laboratorio de Investigaciones en Parasitología Tropical, Facultad de Ciencias, Universidad del Tolima, \\ Ibagué, Colombia. \\ ${ }^{2}$ Centro de Investigaciones en Microbiología y Parasitología Tropical, Universidad de los Andes, Bogotá \\ D.C., Colombia.
}

Introducción. La electroforesis de hemeproteínas salivares en geles de almidón es una técnica de reciente aplicación para diferenciar especies del género Rhodnius que poseen gran similaridad fenotípica o para diferenciar subpoblaciones de la misma especie, separadas geográficamente. De las 15 especies de Rhodnius descritas en latinoamérica, por lo menos ocho han sido reportadas en el territorio colombiano.

Objetivo. Utilizar la electroforesis de hemeproteínas salivares para diferenciar $R$. prolixus de $R$. colombiensis, dos especies con similaridad fenotípica cuyos ciclos doméstico y selvático se superponen en la cuenca alta del río Magdalena en la región Central de Colombia.

Materiales y Métodos. El contenido de las glándulas salivares de cada insecto se sometió a electroforesis en gel de almidón utilizando buffer de glicina y revelado de las bandas con 3,3',5,5'-tetrametilbenzidina. Los patrones de bandeo fueron registrados fotográficamente.

Resultados. Usando esta técnica se observaron los perfiles electroforéticos de las hemeproteínas salivares de $R$. prolixus y $R$. colombiensis los cuales permitieron su inequívoca diferenciación.

Conclusión. Estos resultados demuestran la utilidad de la técnica para la diferenciación entre $R$. prolixus y $R$. colombiensis en adición a métodos morfométricos y moleculares previamente utilizados para diferenciar estas dos especies.

Palabras clave: Rhodnius, Triatominae, saliva, Colombia.

Electrophoretic patterns of salivary hemeproteins (nitrophorines) of Rhodnius colombiensis and $R$. prolixus (Hemiptera, Reduviidae, Triatominae)

Introduction. Salivary hemeprotein electrophoresis in starch gels is a recent technique used for differentiation of Rhodnius species with great phenotypic similarity. Furthermore, populations of the same species can be differentiated from geographically separated locales. Of the 15 described Rhodnius species in Latin America, at least eight have been reported in Colombia. Objective. To use the salivary hemeproteins electrophoresis for $R$. prolixus and $R$. colombiensis identification. These two species are phenotypically similar and have overlapping domestic and sylvatic cycles where they occur in the upper basin of the Magdalena river, Central Colombia. Material and methods. The content of salivary glands of each insect was subjected to starch gel electrophoresis using glycine buffer, and the bands were revealed with 3,3',5,5'tetramethylbenzidine. Band patterns were photographically recorded.

Results. Electrophoretic patterns of salivary hemeproteins of $R$. prolixus and R. colombiensis were able to unequivocally differentiate the two species.

Conclusion. The usefulness of the starch gel technique for distinguishing between $R$. prolixus and $R$. colombiensis was demonstrated as an additional tool to the morphometric and molecular methods already in use for differentiation of these two species.

Key words: Rhodnius, Triatominae, saliva, Colombia. 
La saliva de los insectos hematófagos presenta sustancias anticoagulantes, antihistamínicas, vasodilatorias y antiplaquetarias que facilitan el proceso de alimentación sobre el huésped vertebrado (1-4). En los triatominos se han encontrado varias sustancias relacionadas con estas funciones. En el caso del género Rhodnius, hasta el momento se han reportado ocho proteínas salivares: tres factores que contrarrestan la agregación plaquetaria, llamados inhibidores de agregación de $R$. prolixus (RPAl 1-3) (proteínas lipocalinas) $(4,5)$; una apirasa que se encarga de hidrolizar el ADP bloqueando la agregación plaquetaria $(4,6), y$, por último, las nitroforinas (NPs), un grupo de cuatro hemoproteínas denominadas NP1 a NP4 según su relativa abundancia en las glándulas salivares (7). Las nitroforinas se pueden agrupar en dos conjuntos con base en las relaciones de sus secuencias de aminoácidos; es así como NP1 y NP4 son idénticas en un $90 \%$, y NP2 y NP3 son idénticas en un $80 \%(4,7,8)$. Para Cimex lectularius de la familia Cimicidae se ha reportado otra nitroforina que, aun cuando no es significativamente similar a las de R. prolixus (familia Reduviidae), revelaría una evolución convergente entre estas hemoproteínas $(9,10)$. En Rhodnius, las NP transportan óxido nítrico e histamina a través de su grupo hemo (Fe III), el cual es el sitio de enlace para ambas moléculas $(4,11-14)$. El óxido nítrico permite la vasodilatación e inhibe la agregación plaquetaria $(4,11,15)$. Por su parte, la unión de la histamina a las NP impide la respuesta de inflamación en el huésped vertebrado $(4,12,13)$. La NP2 o prolixin-S se encarga de bloquear la cascada de coagulación, ya que evita que el factor $X$ se convierta en el factor Xa $(4,13,16)$. Por otro lado, se ha comprobado que las NP en $R$. prolixus son estadio específicas, es decir, que puede presentarse una o faltar otra de un estadio a otro. Es así como la NP2 está presente desde el primer

\footnotetext{
Correspondencia:

Gustavo Adolfo Vallejo, Laboratorio de Investigaciones en Parasitología Tropical, Facultad de Ciencias, Universidad del Tolima, Ibagué, Colombia.

Fax (+57-8) 2669176.

gvallejo@ut.edu.co
}

Recibido: 23/03/06; aceptado: 08/09/06 estadio ninfal (N1) hasta el adulto. En el primer estadio se han purificado, además, dos hemoproteínas salivares, la NP5 y la NP6, cuya cantidad va disminuyendo en las glándulas salivares con las mudas sucesivas hasta ausentarse en la fase adulta. En el segundo estadio (N2) aparece la NP4. En el tercer estadio ninfal (N3) se presenta la NP1. Por ultimo, en el quinto estadio ninfal (N5) aparece la NP3, y a partir de este momento ya están presentes las cuatro nitroforinas, NP1 a NP4, que perduran en el adulto (17).

En el género Rhodnius existen varias especies epidemiológicamente importantes para la transmisión de los parásitos $T$. cruzi y $T$. rangeli. Según las características biológicas, ecológicas y etológicas de estos vectores, que suelen variar de una zona geográfica a otra, pueden considerarse como vectores primarios o secundarios, o estar en proceso de alcanzar este último nivel. Algunas especies del género Rhodnius suelen ser fenotípicamente similares y, además, ser simpátricas en algunas zonas geográficas. En los estudios de la epidemiología y la biología de los vectores y de los ciclos de transmisión de los parásitos $T$. cruzi y $T$. rangeli, así como en las encuestas entomológicas y en las campañas de control y prevención vectorial, se han utilizado diferentes tipos de técnicas que han permitido distinguir la mayoría de las especies pertenecientes a este género, entre ellos la morfología (18-20), las isoenzimas (21-23), la morfometría cuantitativa (23), el análisis cuantitativo de patrones de sensilias antenales (24), el análisis de secuencias de ADN mitocondrial $(25,26)$ y el análisis de genitalia masculina $(20,27)$; sin embargo, algunas veces no suelen ser muy efectivas, ya que no generan diferencias $y$, además, algunas son dispendiosas y muy costosas.

Mediante la electroforesis de hemoproteínas salivares es posible diferenciar especies fenotípicamente similares o poblaciones de una misma especie separadas por áreas geográficas $(28,29)$. Además, los perfiles electroforéticos de las hemoproteínas pueden reflejar distancias biogeográficas entre poblaciones y revelar relaciones filogenéticas entre las especies (28). 
Estas diferencias son considerablemente importantes para la epidemiología de los vectores de $T$. cruzi (30) y $T$. rangeli, y ayudan a identificar las especies relacionadas en el ciclo de transmisión de un área geográfica específica. Trabajos previos han mostrado que la diferenciación morfológica de $R$. colombiensis y $R$. prolixus no es fácil, tanto así que durante varios años a la especie $R$. colombiensis se le denominó R. prolixus "forma Tolima" (31); posteriormente, a partir de estudios de isoenzimas, genitalia y genes ribosomales, se concluyó que se trataba de una especie diferente hoy denominada como $R$. colombiensis $(20,32)$. En el presente trabajo se detectaron diferencias por medio de los patrones electroforéticos de hemoproteínas entre las especies $R$. colombiensis y $R$. prolixus, dos especies fenotípicamente similares y simpátricas, ya que sus ciclos selvático y doméstico se traslapan en la cuenca alta del Río Magdalena. Estas especies se han considerado vectores importantes para la transmisión de $T$. cruzi y $T$. rangeli en esa zona geográfica. $R$. colombiensis presenta prevalencias de $T$. cruzi y $T$. rangeli del 45 y $28,6 \%$, respectivamente (datos no publicados), situación que configura un riesgo para la transmisión de $T$. cruzi por parte de este vector en la región central de Colombia.

\section{Materiales y métodos}

\section{Insectos}

Los triatominos fueron recolectados en el municipio de Coyaima, departamento del Tolima, Colombia. Los adultos de $R$. colombiensis fueron capturados en siete palmas de vino (Attalea butyraceae) y los de $R$. prolixus dentro de 10 viviendas humanas de las veredas Totarco Dinde y Chenche Cucal del municipio de Coyaima. Los insectos se mantuvieron a $28 \pm 1^{\circ} \mathrm{C}$, con humedad relativa de 75 a $80 \%$ y una fotoperiodicidad de 12/12 horas en una incubadora automática B.O.D. Lab-Line, y fueron alimentados semanalmente sobre gallinas.

Para la determinación de las especies se utilizó la clave taxonómica de Lent \& Wygodzinsky (1979) (18) y se realizó amplificación por reacción en cadena de la polimerasa (PCR) de genes ADNr siguiendo la metodología descrita por Jaramillo et al. 2001 (32).

\section{Preparación de la muestra y electroforesis}

Se disectaron 13 insectos adultos de $R$. prolixus y 13 de $R$. colombiensis y sus glándulas salivares fueron lavadas en solución salina estéril al 0,9\%. Las glándulas salivares de cada insecto se maceraron y homogeneizaron con $10 \mathrm{ml}$ de buffer de corrido (glicina 0,15 M/NaOH, pH 9,5). Una vez homogeneizadas, el contenido de las glándulas fue absorbido en hilos de algodón estériles de 0,9 $\mathrm{cm}$ y colocados en las canaletas del gel de almidón al 8\% preparado con buffer de corrido (glicina 0,15M/NaOH, pH 9,5) diluido 1:10. La electroforesis se llevó a cabo a 300 voltios por cuatro horas. Para el revelado, los geles se sumergieron en una solución de $0,3 \mathrm{mg} / \mathrm{ml}$ de 3,3',5,5'-tetrametilbenzidina en una mezcla de etanol, ácido acético y agua en proporción 1:1:1. Luego se transfirieron a una solución de peróxido de hidrógeno al $2 \%$ hasta la aparición de las bandas $(28,29)$. Con fines comparativos, se examinaron los patrones electroforéticos de las hemoproteínas de dos adultos de R. pallescens.

El número de bandas generado en cada carril del gel fue determinado en el momento inicial en que se desarrolla la coloración, pues a medida que transcurre el tiempo, las bandas pierden resolución. A partir de las fotografías y del número de bandas observadas al inicio de la coloración se obtuvieron diagramas que representan el número de bandas en cada carril.

\section{Análisis de datos}

De conformidad con el análisis propuesto por Soares et al. (29), se calculó el índice de polimorfismo de hemoproteínas $(P)$ para cada especie, $p=1-\Sigma\left(X^{2}\right)$, donde $X$ es igual a la frecuencia absoluta de cada perfil de hemoproteínas en la especie sobre el número total de insectos examinados.

\section{Resultados}

El número de perfiles y el patrón de bandeo obtenido a través de la electroforesis de hemoproteínas salivares en geles delgados de almidón para $R$. colombiensis y $R$. prolixus 
permitió establecer una clara diferencia entre las dos especies (figura 1 y 2). R. prolixus presentó cinco perfiles en el corrido electroforético, designados desde la $A$ hasta la $E$ en las figuras 1 y 2; por otro lado, $R$. colombiensis mostró tres perfiles (A, B y C) (figuras 1 y 2 )

$R$. prolixus presentó un promedio de 3,1 bandas, con un máximo de cinco y un mínimo de dos; en $R$. colombiensis solamente se observaron dos bandas en todos las insectos examinados (figuras 1 y 2). Además, se observó que las hemoproteínas salivares de $R$. colombiensis tienen polaridad negativa, pues corrieron hacia el polo positivo. En R. prolixus, las hemoproteínas presentan carga positiva y negativa, pues en el corrido electroforético fue evidente una atracción hacia ambos polos (figura 1 y 2 ).

El índice de polimorfismo de las hemoproteínas salivares $(p)$ para las dos especies estudiadas fue de $p=0,7577$ en $R$. prolixus y en $R$. colombiensis de $p=0,6156$.

\section{Discusión}

R. prolixus presentó un índice de polimorfismo de hemoproteínas $(p)$ alto $(p=0,7577)$ con respecto a $R$. colombiensis, que presentó un índice inferior
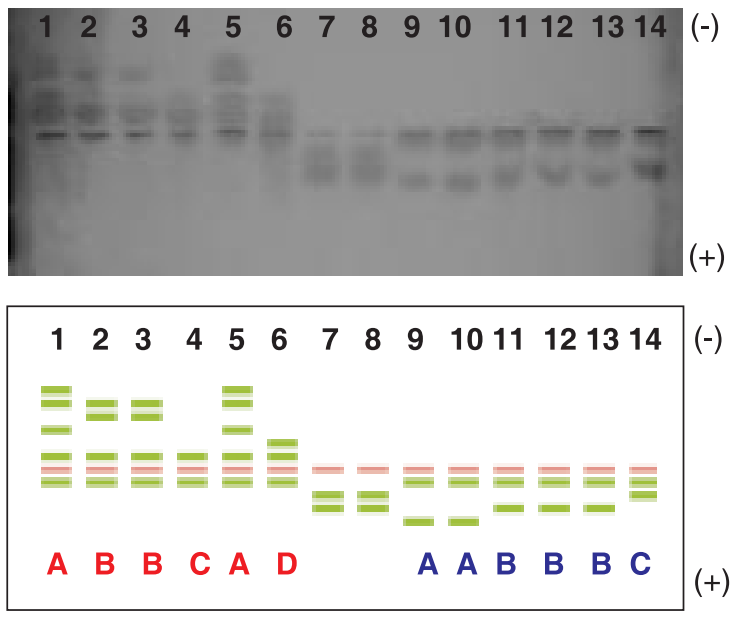

Figura 1. Electroforesis en gel delgado de almidón al $8 \%$ de hemoproteínas salivares de $R$. prolixus (canaletas 1 a 6 ), $R$. pallescens (canaletas 7 y 8 ) y $R$. colombiensis (canaletas 9 a 14). El panel inferior de la figura corresponde a un diagrama que representa las bandas del panel superior.
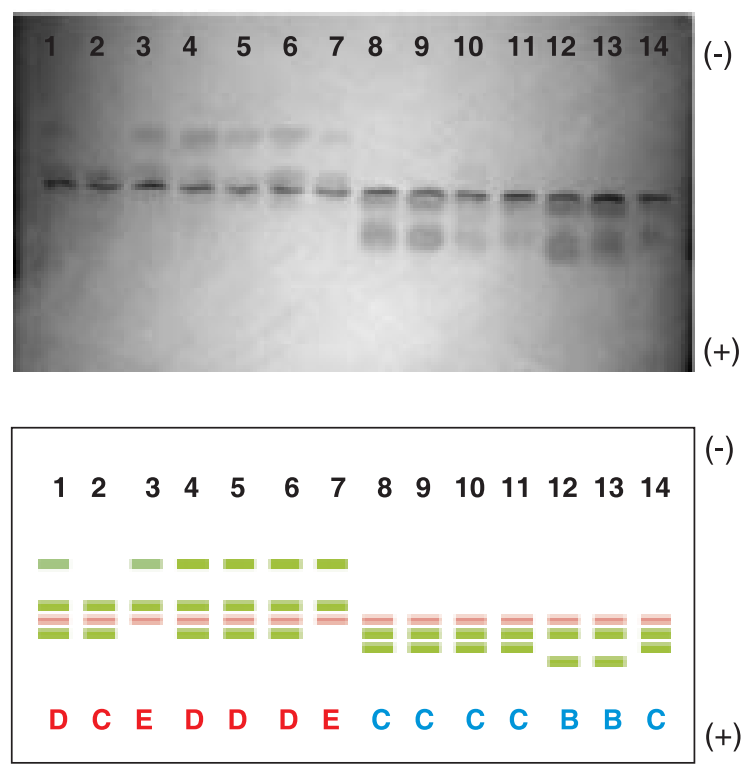

Figura 2. Electroforesis en gel delgado de almidón al 8\% de hemoproteínas salivares de $R$. prolixus (canaletas 1 a 7) y R. colombiensis (canaletas 8 a 14). El panel inferior de la figura corresponde a un diagrama que representa las bandas del panel superior.

$(p=0,6156)$. Para Soares et al., (29) el índice de polimorfismo de $R$. prolixus presentó un valor $p=$ 0,7300 , inferior en 0,0277 al valor obtenido para esta especie $(p=0,7577)$ en el presente trabajo. Es probable que esta diferencia se deba a la procedencia de ambas poblaciones, ya que la población utilizada por Soares et al. (29) era originaria de Venezuela, mientras que los $R$. prolixus utilizados en el presente estudio se recolectaron en la región central de Colombia. Por otro lado, los perfiles electroforéticos de las hemoproteínas pueden reflejar distancias biogeográficas entre las poblaciones estudiadas (28). El valor $(p)$ de $R$. colombiensis $(p=0,6156)$ se acerca mucho al índice de polimorfismo de $R$. pallescens ( $p=0,6524)$ (29), especies éstas morfológicamente similares $(20,31)$. Estos valores estarían reflejando el parentesco filogenético entre estas dos especies pertenecientes al grupo "pallescens", conformado por $R$. pallescens, $R$. colombiensis y $R$. ecuadoriensis que representa un cline evolutivo desde el norte de Colombia hasta el sur de la cordillera de los Andes, en donde finalmente se originó $R$. ecuadoriensis en el Ecuador y norte del Perú (31). 
La electroforesis de hemoproteínas salivares es una técnica fácil y rápida que permite diferenciar especies del género Rhodnius, al cual pertenecen especies epidemiológicamente significativas en la transmisión de los parásitos $T$. cruzi y $T$. rangeli en muchas regiones de Colombia. Además, la técnica podría ser útil en casos en que no se pueda diferenciarlas debido a la similitud morfológica de las especies.

Por otro lado, podría ser una herramienta útil para los trabajos de encuestas entomológicas y en los programas de control y prevención vectorial, pues mediante ella se podrían identificar las especies de una manera fácil y rápida cuando se encuentren circulando en los ciclos de transmisión silvestre o doméstico en una región geográfica específica. Ello también sería útil cuando se van a emplear estrategias de control vectorial, ya que pueden existir especies silvestres que estén invadiendo las viviendas, como en el caso de $R$. colombiensis en la cuenca alta del río Magdalena (región central de Colombia), cuyas ninfas de cuarto, quinto estadio, hembras y machos han sido encontradas durante trabajos previos en viviendas y construcciones peridomiciliares de zonas en donde se adelantan programas de control tendientes a eliminar a $R$. prolixus, el principal vector domiciliado en Colombia (datos no publicados). La técnica de electroforesis de hemoproteínas es una herramienta que permite diferenciar especies de triatominos de una forma fácil, sin embargo, es importante durante el procedimiento de coloración registrar inmediatamente el número original de las bandas, pues la intensidad de las mismas va disminuyendo con el tiempo. En conclusión, estos resultados demuestran la utilidad de la técnica para la diferenciación entre $R$. prolixus y $R$. colombiensis y como complemento de métodos morfométricos y moleculares previamente utilizados para diferenciarlas.

\section{Conflicto de intereses}

El primer autor y los coautores del presente artículo declaramos que no teníamos conflictos de intereses de orden académico, institucional u operacional en el momento de realización de la investigación.

\section{Financiación}

Este trabajo recibió financiación del Instituto Colombiano "Francisco José de Caldas" (Colciencias), proyecto 105-05-279-99, y del Fondo de Investigaciones de la Universidad del Tolima. También recibió apoyo internacional de la Red ECLAT (European Community-Latin-American Network for Research on the Biology and Control of Triatominae).

\section{Referencias}

1. Stark KR, James AA. The salivary glands of disease vectors. En: Beaty BJ, Marquardt WC, editors. The biology of disease vectors. Colorado: Colorado University Press; 1996. p. 333-48.

2. Ribeiro JM. Role of saliva in blood feeding by arthropods. Annu Rev Entomol 1987;32:463-78.

3. Law JH, Ribeiro JM, Wells MA. Biochemical insights derived from diversity in insects. Annu Rev Biochem 1992;61:87-111.

4. Montfort WR, Weichsel A, Andersen JF. Nitrophorins and related antihemostatic lipocalins from Rhodnius prolixus and other blood-sucking arthropods. Biochim Biophys Acta 2000;1482:110-8.

5. Francischetti IM, Ribeiro JM, Champagne D, Andersen J. Purification, cloning, expression and mechanism of action of a novel platelet aggregation inhibitor from salivary gland of the blood-sucking bug, Rhodnius prolixus. J Biol Chem 2000;275:12639-50.

6. Sarkis JJ, Guimarães JA, Ribeiro JM. Salivary apyrase of Rhodnius prolixus. Kinetics and purification. Biochem J 1986;233:885-91.

7. Champagne DE, Nussenzveig RH, Ribeiro JM. Purification, partial characterization and cloning of nitric oxide-carrying hemeproteins (nitrophorins) from salivary glands of the blood-sucking insect Rhodnius prolixus. J Biol Chem 1995;270:8691-5.

8. Andersen JF, Montfort WR. The crystal structure of Nitrophorin 2. A trifunctional antihemostatic protein from the saliva of Rhodnius prolixus. J Biol Chem 2000;275:30496-503.

9. Valenzuela JG, Walker FA, Ribeiro JM. A salivary nitrophorin (nitric-oxide-carrying hemoprotein) in the bedbug Cimex lectularius. J Exp Biol 1995;198:1519-26.

10. Valenzuela JG, Ribeiro JM. Purification and cloning of the salivary nitrophorin from the hemipteran Cimex lectularius. J Exp Biol 1998;201:2659-64.

11. Ribeiro JM, Hazzard JM, Nussenzveig RH, Champagne DE, Walker FA. Reversible binding of nitric oxide by a salivary heme protein from a bloodsucking insect. Science 1993;260:539-41. 
12. Ribeiro JM, Walker FA. High affinity histamine-binding and antihistaminic activity of the salivary nitric oxidecarrying heme protein (nitrophorin) of Rhodnius prolixus. J Exp Med 1994;180:2251-7.

13. Ascenzi P, Nardini M, Bolognesi M, Montfort WR. Nitrophorins. Lipocalin-based heme proteins transporting nitric oxide. Biochem Mol Biol Educ 2002;30:68-71.

14. Walker FA. Nitric oxide interaction with insect nitrophorins and thoughts on the electron configuration of the $\left\{\mathrm{FeNO}^{6}\right.$ complex. J Inorg Biochem 2005;99: 216-36.

15. Moncada S, Radomski MW, Palmer RM. Endotheliumderived relaxing factor: identification as nitric oxide and role in the control of vascular tone and platelet function. Biochem Pharmacol 1988;37:2495-501.

16. Ribeiro JM, Schneider M, Guimarães JA. Purification and characterization of prolixin $S$ (nitrophorin 2), the salivary anticoagulant of the blood-sucking bug Rhodnius prolixus. Biochem J 1995;308:243-9.

17. Moreira FM, Coelho HS, Zingali RB, Oliveira PL, Masuda $\mathbf{H}$. Changes in salivary nitrophorin profile during the life cycle of the blood-sucking bug Rhodnius prolixus. Insect Biochem Mol Biol 2003;33:23-8.

18. Lent H, Wygodzinsky P. Revision of Triatominae (Hemiptera, Reduviidae), and their significance as vectors of Chagas' disease. Bull Am Mus Nat Hist 1979;163:123-520.

19. Carcavallo R, Galíndez I, Jurberg J, Lent H. Atlas of Chagas' disease vectors in the Americas. Río de Janeiro: Editora Fiocruz; 1998. p.53-72.

20. Moreno J, Galvão C, Jurberg J. Rhodnius colombiensis $s p$. $n$. da Colômbia com quadros comparativos entre estructuras fálicas do género Rhodnius Stal. 1859 (Hemiptera, Reduviidae, Triatominae). Entomol Vect 1999;6:601-17.

21. Harry M, Galíndez I, Cariou ML. Isoenzyme variability and differentiation between Rhodnius prolixus, $R$. robustus and $R$. pictipes, vectors of Chagas disease in Venezuela. Med Vet Entomol 1992;6:37-43.

22. Chavez T, Moreno J, Dujardin JP. Isoenzyme electrophoresis of Rhodnius species: a phenetic approach to relationships within the genus. AnnTrop Med Parasitol 1999;93:299-307.
23. Dujardin JP, Chavez T, Moreno JM, Machane M, Noireau F, Schofield CJ. Comparison of izoenzyme electrophoresis and morphometric analysis for phylogenetic reconstruction of the Rhodniini (Hemiptera: Reduviidae: Triatominae). J Med Entomol 1999;36:653-9.

24. Catalá S, Schofield C. Antennal sensilla of Rhodnius. J Morphol 1994;219:193-203.

25. Stothard JR, Yamamoto Y, Cherchi A, Garcia AL, Valente SA, Schofield CJ, et al. A preliminary survey of mitochondrial sequence variation in Triatominae (Hemiptera: Reduviidae) using polymerase chain reaction-based single strand conformational polymorphism (SSCP) analysis and direct sequencing. Bull Entomol Res 1998;88:553-60.

26. Lyman DF, Monteiro FA, Escalante AA, CordonRosales C, Wesson DM, Dujardin JP, et al. Mitochondrial DNA sequence variation among triatomine vectors of Chagas disease. Am J Trop Med Hyg 1999;60:377-86.

27. Jurberg J. Uma abordagem filogenética entre os Triatomineos baseada nas estruturas fálicas. In: Schofield CJ, Dujardin JP, Jurberg J, editors. Proceedings of the International Workshop on Population Biology and Control of Triatominae, Santo Domingo de los Colorados, Ecuador. Mexico D.F: INDRE; 1996. p.45-50.

28. Soares RP, Gontijo NF, Romanha AJ, Diotaiuti L, Pereira MH. Salivary heme proteins distinguish Rhodnius prolixus from Rhodnius robustus (Hemiptera: Reduviidae: Triatominae). Acta Trop 1998;71:285-91.

29. Soares RP, Sant'Anna MR, Gontijo NF, Romanha AJ, Diotaiuti L, Pereira MH. Identification of morphologically similar Rhodnius species (Hemiptera: Reduviidae: Triatominae) by electrophoresis of salivary heme proteins. Am J Trop Med Hyg 2000;62:157-61.

30. WHO. Control of Chagas' disease. Geneve: World Health Organ Tech Rep Ser. 811; 1991. p.95.

31. Schofield CJ, Dujardin JP. Theories on the evolution of Rhodnius. Actualidades Biológicas 1999;21:183-97.

32. Jaramillo C, Montaña MF, Castro LR, Vallejo GA, GuhI F. Differentiation and genetic analysis of Rhodnius prolixus and Rhodnius colombiensis by rDNA and RAPD amplification. Mem Inst Oswaldo Cruz 2001;96:1043-8. 